\title{
Characterization of dengue cases among patients with an acute illness, Central Department, Paraguay
}

\author{
Alejandra Rojas ${ }^{\text {Corresp., } 1}{ }^{\text {, Fátima Cardozo }}{ }^{2}$, César Cantero ${ }^{1}$, Victoria Stittleburg ${ }^{3}$, Sanny López ${ }^{1}$, Cynthia Bernal ${ }^{1}$, \\ Fracisco Giménez $^{4}$, Laura Mendoza ${ }^{2}$, Benjamin A Pinsky ${ }^{5,6}$, Yvalena Guillén ${ }^{1}$, Malvina Páez ${ }^{2}$, Jesse Waggoner ${ }^{\text {Corresp. }}$ \\ 3,7

Background. In 2018, Paraguay experienced a large dengue virus (DENV) outbreak. The primary objective of this study was to characterize dengue cases in the Central Department, where the majority of cases occur, and identify factors associated with DENV infection. Methods. Patients were enrolled from January-May 2018 if they presented with a suspected arboviral illness. Acute-phase specimens ( $\leq 8$ days after symptom onset) were tested using rRT-PCR, a rapid diagnostic test for DENV nonstructural protein 1 (NS1) and anti-DENV IgM and IgG, and ELISA for IgG against NS1 from Zika virus (ZIKV). Results. 231 patients were enrolled (95.2\% adults) at two sites: emergency care and an outpatient clinical site. Patients included 119 (51.5\%) dengue cases confirmed by rRT-PCR ( $n=115$, $96.6 \%)$ and/or the detection of NS1 and anti-DENV IgM ( $n=4,3.4 \%)$. DENV-1 was the predominant serotype (109/115, 94.8\%). Epidemiologically, dengue cases and non-dengue cases were similar, though dengue cases were less likely to reside in a house/apartment or report a previous dengue case. Clinical and laboratory findings associated with dengue included red eyes, absence of sore throat, leucopenia and thrombocytopenia. At an emergency care site, $26 \%$ of dengue cases (26/100) required hospitalization. In univariate analysis, hospitalization was associated with increased viral load, anti-DENV IgG, and thrombocytopenia. Among dengue cases that tested positive for IgG against ZIKV NS1, the odds of DENV NS1 detection in the acute phase were decreased 10-fold (OR 0.1, 0.0-0.3). Conclusions. Findings from a predominantly adult population demonstrate clinical and laboratory factors associated with DENV infections and the potential severity of dengue in 
this group. The combination of viral load and specific IgG antibodies warrant further study as a prognostic to identify patients at risk for severe disease. 
1 Characterization of dengue cases among patients with an acute illness, Central

2 Department, Paraguay

3

4 Alejandra Rojas,,${ }^{1,}$ Fátima Cardozo, ${ }^{2}$ César Cantero, ${ }^{1}$ Victoria Stittleburg, ${ }^{3}$ Sanny López, ${ }^{1}$

5 Cynthia Bernal, ${ }^{1}$ Francisco Giménez, ${ }^{4}$ Laura Mendoza, ${ }^{2}$ Benjamin A. Pinsky, ${ }^{5,6}$ Yvalena

6 Guillén, ${ }^{1}$ Malvina Páez, ${ }^{2}$ Jesse J. Waggoner ${ }^{3,7,}$,

7

$8{ }^{1}$ Universidad Nacional de Asunción, Instituto de Investigaciones en Ciencias de la Salud,

9 Departamento de Producción, Paraguay

$10{ }^{2}$ Universidad Nacional de Asunción, Instituto de Investigaciones en Ciencias de la Salud,

11 Departamento de Salud Pública, Paraguay

$12{ }^{3}$ Department of Medicine, Division of Infectious Diseases, Emory University, Atlanta, Georgia,

13 United States

$14{ }^{4}$ Área Ambulatoria, Hospital Distrital de Villa Elisa, Asunción, Paraguay

$15{ }^{5}$ Department of Pathology, Stanford University School of Medicine, Stanford, California, United

16 States

$17{ }^{6}$ Department of Medicine, Division of Infectious Diseases and Geographic Medicine, Stanford

18 University School of Medicine, Stanford, California, United States

$19{ }^{7}$ Rollins School of Public Health, Department of Global Health, Emory University, Atlanta,

20 Georgia, United States

21

22 * Corresponding Authors: Alejandra Rojas, email: arojass@iics.una.py; Jesse J. Waggoner,

23 email: jesse.j.waggoner@emory.edu 
24 Abstract

25 Background. In 2018, Paraguay experienced a large dengue virus (DENV) outbreak. The primary objective of this study was to characterize dengue cases in the Central Department, where the majority of cases occur, and identify factors associated with DENV infection.

Methods. Patients were enrolled from January-May 2018 if they presented with a suspected arboviral illness. Acute-phase specimens ( $\leq 8$ days after symptom onset) were tested using rRTPCR, a rapid diagnostic test for DENV nonstructural protein 1 (NS1) and anti-DENV IgM and IgG, and ELISA for IgG against NS1 from Zika virus (ZIKV).

Results. 231 patients were enrolled (95.2\% adults) at two sites: emergency care and an outpatient clinical site. Patients included 119 (51.5\%) dengue cases confirmed by rRT-PCR $(n=115,96.6 \%)$ and/or the detection of NS1 and anti-DENV IgM $(n=4,3.4 \%)$. DENV-1 was the predominant serotype (109/115, 94.8\%). Epidemiologically, dengue cases and non-dengue cases were similar, though dengue cases were less likely to reside in a house/apartment or report a previous dengue case. Clinical and laboratory findings associated with dengue included red eyes, absence of sore throat, leucopenia and thrombocytopenia. At an emergency care site, $26 \%$ of dengue cases $(26 / 100)$ required hospitalization. In univariate analysis, hospitalization was associated with increased viral load, anti-DENV IgG, and thrombocytopenia. Among dengue cases that tested positive for IgG against ZIKV NS1, the odds of DENV NS1 detection in the acute phase were decreased 10-fold (OR 0.1, 0.0-0.3). 
46 Conclusions. Findings from a predominantly adult population demonstrate clinical and

47 laboratory factors associated with DENV infections and the potential severity of dengue in this

48 group. The combination of viral load and specific IgG antibodies warrant further study as a

49 prognostic to identify patients at risk for severe disease. 
50

51

52

53

54

55

56

57

58

59

60

61

62

63

64

65

66

67

68

69

70

71

72

\section{Introduction}

Dengue is the commonest human arboviral disease worldwide, with an estimated 50-100 million cases occurring annually throughout the tropics and subtropics (Stanaway et al. 2016; World Health Organization 2009). Dengue results from human infection with one of four related serotypes of dengue virus (DENV-1-4) (Guzman \& Harris 2015). In the five years leading up to and including the current study (2018), all four serotypes circulated in the region of South America surrounding Paraguay (Fig. 1), which reports among the highest annual incidence rates of dengue on the continent (Dantes et al. 2014; Gordon et al. 2013; Pan American Health Organization 2018). Over the past decade, DENV-1 has circulated in Paraguay in all but one year, and it has been predominant since 2015 (Dirección General de Vigilancia de la Salud \& Ministerio de Salud Pública y Bienestar Social 2017; Pan American Health Organization 2018). Despite significant declines in dengue incidence throughout the Americas following the 20152016 Zika virus (ZIKV) epidemic, Paraguay experienced large numbers of dengue cases in 2016 and again in 2018 (Pan American Health Organization 2018; Perez et al. 2019). These data suggest that arboviral epidemiology may be relatively unique in Paraguay, which is located at the southern boundary of the DENV-endemic region in the Americas (Bhatt et al. 2013; Stanaway et al. 2016; World Health Organization 2009). However, relatively little data has been published on dengue in the country, and the majority of available data has come either from hospitalized pediatric cases or from international studies with only a subset of patients from Paraguay (Halsey et al. 2012; Lovera et al. 2014; Lovera et al. 2016; Rojas et al. 2016).

Symptomatic DENV infections classically present as an acute fever with myalgias and rash (Guzman \& Harris 2015; World Health Organization 2009). However, patients can develop a 
73 wide array of signs and symptoms, which limits the accuracy of a clinical diagnosis based on

74 exam findings and results of routine laboratory testing (Gregory et al. 2010; Morch et al. 2017;

75 Potts \& Rothman 2008; Waggoner et al. 2016b). In addition, dengue manifests differently among

76 children and adults, and factors associated with dengue cases and severe disease in a pediatric

77 population may not be applicable in older patients (Gregory et al. 2010; Hammond et al. 2005;

78 Kittigul et al. 2007). The differential diagnosis for dengue includes arboviral pathogens, such as

79 chikungunya virus (CHIKV) and Zika virus (ZIKV), and local endemic diseases, such as

80 leptospirosis, which may all cause an indistinguishable clinical picture (O. Silva et al. 2018;

81 Waggoner et al. 2016b). Accurate diagnosis in the acute phase relies upon the availability of specific laboratory tests, which for DENV include molecular methods and nonstructural protein 1 (NS1) antigen detection. Anti-DENV IgM detection in acute-phase samples provides a presumptive diagnosis (Peeling et al. 2010; World Health Organization 2009). As dengue can progress to severe disease, including plasma leakage, hemorrhage, and shock, ideal testing algorithms would not only detect DENV infections but also provide prognostic information.

The primary objective of the current study was to characterize DENV infections in the Central Department of Paraguay and the metropolitan area of Asunción. This region annually accounts for $\sim 2 / 3$ of dengue cases in Paraguay and also reported Zika cases in 2016. Multiplex molecular testing, NS1 antigen detection and serological methods were implemented to confirm cases identified using a broad clinical case definition. We then sought to evaluate factors associated with dengue cases and the need for hospitalization in a predominantly adult patient population.

\section{Materials \& Methods}


97 Ethics statement. The study protocol was reviewed and approved by the Scientific and Ethics

Committee of the Instituto de Investigaciones en Ciencias de la Salud, Universidad Nacional de

Asunción (IICS-UNA, IRB00011984), and the Emory University Institutional Review Board

(IRB00000569). Written informed consent was obtained from all subjects. Children older than

101 six years of age provided assent.

102

Patient population and clinical samples. Patients of all ages were enrolled from January to

May 2018 if they presented with an acute illness ( $\leq 8$ days) defined by 2 or more of the

following: fever (measured or subjective), red eyes, rash, joint pain involving more than one joint, and/or diffuse muscle pain. Patients with fever and no other localizing signs or symptoms were included. Day 1 was defined as the first day of symptoms. Exclusion criteria included dysuria or malodorous urine, cellulitis/skin abscess, vomiting and/or a productive cough. Patients were enrolled at in the Emergency Care Clinic at Hospital Villa Elisa and at IICS-UNA, both located in metro Asunción. The Emergency Care Clinic serves an ambulatory urgent care patient population; patients may be assigned to observation at Hospital Villa Elisa or referred to an inpatient facility that can provide a higher level of care. Serum was collected during the acute visit, aliquoted and stored at $-80^{\circ} \mathrm{C}$ until use. The results from hemograms, performed as part of routine care, were obtained by chart review. Data was included in this study if the hemogram was obtained on the day of the study visit \pm 1 day.

Molecular detection. RNA was extracted from $140 \mu \mathrm{L}$ of serum into $60 \mu \mathrm{L}$ of elution buffer with the QIAamp Viral RNA Mini Kit (Qiagen, Germantown, MD). All samples were tested for 
119 ZIKV, CHIKV and DENV by real-time RT-PCR (rRT-PCR) using a validated and published

120 multiplex assay (the ZCD assay) as previously described (Waggoner et al. 2016a). DENV

121 serotype and viral load were determined with a DENV multiplex assay using a published

122 protocol (Waggoner et al. 2013b; Waggoner et al. 2013c). Samples that tested negative in the

123 ZCD assay were tested for RNase P to confirm successful extraction and the absence of

124 inhibitors (Waggoner et al. 2013a). All rRT-PCR testing was performed at IICS-UNA.

Serological assays. All serum samples were test for DENV NS1 antigen and anti-DENV IgM

and IgG using the STANDARD Q Dengue Duo assay (SD Biosensor, Suwon, South Korea).

Results were read initially at 15 or up to 20 minutes, according to manufacturer

recommendations. One hundred fifty-six samples were tested for anti-ZIKV IgG using the the ZIKV NS1 antigen. Given a limited supply of anti-ZIKV IgG kits, a mixture of samples was selected for testing. This included dengue cases $(n=76)$ and non-dengue cases $(n=80)$, as well as include patients with anti-DENV $\operatorname{IgG}(n=58)$ and without $(n=98)$. Assays were performed according to manufacturer recommendations.

Definitions. Dengue cases were defined by either the detection of 1) DENV RNA in serum using 137 the ZCD assay with confirmation in the DENV multiplex assay, or 2) both NS1 and anti-DENV 138 IgM. This conservative definition was used to ensure the accuracy of dengue-case calls in the 139 absence of paired acute and convalescent sera for confirmatory serological testing. This definition also allowed us to evaluate the performance of the STANDARD Q DENV NS1 assay,

141 for which there was no prior published data. The sensitivity and specificity of individual 
142 diagnostics were calculated in reference to positive and negative cases from this composite 143 definition.

144

145 Statistics. Basic statistical analyses were performed using Excel software (Microsoft, Redmond, 146 WA). Univariate analyses and multiple linear regression analyses were performed using

147 GraphPad Prism, version 8.0.1 (GraphPad, San Diego, CA). Categorical variables were 148 compared using Fisher's exact test. Age, day of illness, and continuous laboratory variables were 149 compared by $t$ test. Viral load comparisons were performed using non-parametric tests (Mann150 Whitney with 2 groups; Kruskal-Wallis for 3 or more groups). Binary logistic regression analysis was performed using SPSS (IBM, Armonk, NY). Model fit was assessed by comparing -2 log 152 likelihood statistics.

\section{Results}

Between January and May 2018, we enrolled 231 patients who met inclusion criteria, including $119(51.5 \%)$ dengue cases and 112 (48.5\%) non-dengue cases. No acute cases of ZIKV or CHIKV were detected. Of the dengue cases, 115 (96.6\%) tested positive by rRT-PCR and 4 additional cases (3.4\%) were positive for DENV NS1 and anti-DENV IgM (Table 1). All cases tested positive by rRT-PCR through day-of-illness $6(n=104)$, with rates of detection declining on days 7 (7/10, 70\%) and 8 (4/5, 80\%; Fig. 2). For the NS1 assay, the overall sensitivity and 161 specificity were $71.4 \%$ and $96.4 \%$, respectively (Table 1). Although there appeared to be an 162 increase in NS1 sensitivity over the first 5 days of illness, this was not statistically significant $(p=0.208$, day 5 vs. day 1-2; Fig. 2). The overall sensitivity and specificity of anti-DENV IgM 
164 detection were $26.1 \%$ and $93.8 \%$, respectively. The sensitivity of IgM detection increased from

$1650 \%$ on days $1-2$ to $90 \%$ on day $7(\mathrm{p}<0.001)$.

166

167 DENV serotype was determined in all 115 rRT-PCR-positive cases, with DENV-1 identified in 109/115 (94.8\%) cases and 3 cases (2.6\%) each of DENV-2 and DENV-4. No co-infections were detected. DENV-1 serum viral loads negatively correlated with day of illness at presentation (Fig. 3A), but too few data points were available for DENV-2 and -4 to draw meaningful

171

172

173

174

175

176

177

178

179

180

181

182

183

184

185

conclusions. DENV viral load was also associated with NS1 detection: viral loads were

significantly higher in samples with detectable NS1 (median $7.7 \log _{10}$ copies/mL, IQR 5.8-8.6)

compared to those in which NS1 was not detectable (median 5.6 $\log _{10}$ copies/mL, IQR 3.6-7.2;

$\mathrm{p}<0.001$; Fig. S1).

Anti-DENV and anti-ZIKV IgG results were available for 156 patients, including 76 dengue cases (48.7\%). 58 patients (37.2\%) tested positive for anti-DENV IgG and $49(31.4 \%)$ tested positive for anti-ZIKV IgG, with 32 patients $(20.5 \%)$ positive for both. The viral load among dengue cases declined in a stepwise manner among patients with anti-ZIKV IgG, anti-DENV IgG, or both (Fig. 3B, $\mathrm{p}<0.001$ for the trend). In a multivariable model that included day of illness at presentation and patient age, DENV serum viral load was $1.3 \log _{10}$ copies $/ \mathrm{mL}$ lower among patients with detectable anti-DENV IgG compared to patients without anti-DENV IgG $\left(\mathrm{p}<0.001\right.$, Table S1). Similarly, serum viral load was $0.7 \log _{10}$ copies/mL lower among patients with anti-ZIKV IgG directed against NS1 $(\mathrm{p}=0.047)$.

Peer) reviewing PDF | (2019:06:38824:1:2:NEW 29 Aug 2019) 
186 Among dengue cases, DENV NS1 detection was also associated with IgG status. Patients with 187 anti-DENV IgG were significantly less likely to have detectable NS1 [20/36 (55.6\%) vs. 65/83

188 (78.3\%); OR 0.3, 95\% CI 0.1-0.8]. However, when we controlled for the detection of IgG against 189 ZIKV NS1, the OR for NS1 detection among dengue cases with anti-ZIKV IgG was 0.1 (95\% CI 190 0.0-0.3) and the association with anti-DENV IgG was no longer significant (OR 1.0, 95\% CI 0.3191 3.1; Table S2).

192

193 Epidemiologic characteristics. The epidemiologic characteristics of the patient population are 194 shown in Table 2 . This was predominantly an adult population, with only 11 participants $<18$ 195 years of age at study entry (4.8\%). Dengue cases occurred throughout the study period (Fig. S2) and were similar to non-dengue cases for the majority of epidemiological variables analyzed. Most patients reported living in a house or an apartment (157/188 for which data was available, $83.5 \%$ ), but 31 patients reported "other" for housing without providing further detail. The odds of dengue in this population were significantly higher than among patients with a different living arrangement (OR 2.9, 95\% CI 1.3-7.0). Only 10.6\% of our patients (21/199) reported having screens on their windows, though $79.2 \%$ of patients had air conditioning (156/197). The percentage of dengue cases among patients with neither screens nor air conditioning (19/37, $51.4 \%)$ was similar to that of patients with screens, air conditioning, or both $(79 / 161,49.1 \%$; OR $1.1,95 \%$ CI $0.5-2.2)$.

A subset of patients self-reported having been vaccinated against yellow fever virus (YFV). The odds of having a dengue case were lower among patients who had received the YFV vaccine compared to those who had not (OR 0.6; 0.4-1.2), and more time had elapsed since vaccination 
209

210

211

212

213

214

215

216

217

218

219

220

221

222

223

224

225

226

227

228

229

230

231

among dengue cases. However, these trends did not reach statistical significance $(\mathrm{p}=0.15)$.

Receipt of the YFV vaccine did not increase the need for hospitalization among dengue cases.

Clinical presentation. Patient symptoms at presentation are shown in Table 3. The majority of patients met inclusion criteria with fever plus one additional symptom in the study definition, most commonly muscle pain $(198 / 225,88.0 \%)$ and/or joint pain $(172 / 221,77.8 \%)$. Only 11 patients (4.8\%) had fever and no other localizing sign or symptom (6 dengue cases), and 8 patients $(3.5 \%)$ were enrolled that did not have fever ( 1 dengue case). Patients who reported red eyes were significantly more likely to have dengue (OR 2.1; 95\% CI 1.2-3.6) and those with a sore throat were significantly less likely to have dengue (OR 0.5; 95\% CI 0.3-0.8; Table 3). Although a reported headache increased the odds of having dengue, this did not reach statistical significance (OR 2.3; 95\% CI 1.0-5.5), and headache was very common overall. Other symptoms occurred with similar frequency in the two groups, and no combination of symptoms accurately differentiated between dengue and non-dengue cases.

Hemogram results are also shown in Table 3. Patients with dengue had significantly lower platelet and leucocyte counts relative to non-dengue cases (Fig. 4). Thrombocytopenia $(<150,000$ per $\mu \mathrm{L})$ and leucopenia $\left(<4,000\right.$ cells $\left./ \mathrm{mm}^{3}\right)$ were both significantly associated with DENV infections (Table 3). However, patients with both findings were not at greater odds of having a DENV infection (OR 8.9; 95\% CI 3.4-23.0) than patients with leucopenia alone (OR 11.0, 95\% CI 5.1-22.2). Dengue cases had lower neutrophil and lymphocyte counts, but these occurred in proportion to the decrease in leucocyte counts (see Supplemental Files, Raw Data). 
232 Hospitalization. For the analysis of factors associated with hospitalization for dengue, we

233 focused on cases that presented to Hospital Villa Elisa, as only $1 / 19$ dengue cases (5.3\%) at 234 IICS-UNA required hospitalization. Of 100 dengue cases at Hospital Villa Elisa, 26 (26.0\%)

235 were hospitalized and one patient died (Table 4). A number of clinical and laboratory findings

236 were associated with hospitalization in univariate analysis. Rash and bleeding were more

237 common among hospitalized cases. Admitted patients were significantly more likely to have

238 detectable anti-DENV IgG and IgG against both DENV and ZIKV (anti-NS1). Despite the

239 presence of anti-DENV IgG, viral load was significantly higher among admitted patients, but

240 there was no difference in NS1 detection. In multivariate analysis, the best-fit model for

241 predictors of hospitalization only included platelet count and day of illness, though the odds ratio

242 for day of illness did not reach significance (OR 1.3, 95\% CI 0.9-1.8; Table S3).

\section{Discussion}

245 In the current study, we characterized a set of dengue cases in a primarily adult population that

246 presented to outpatient facilities in metro Asunción. Dengue is a major public health problem in

247 Paraguay, with adults accounting for a significant proportion of cases. At Hospital Villa Elisa,

$24858 \%$ of patients with an acute febrile illness were adults $\geq 20$ years of age, and an additional $13 \%$

249 of patients were aged 15-19. While studies have demonstrated that the clinical presentation of

250 dengue in adults may differ from that in children (Hammond et al. 2005; Kittigul et al. 2007;

251 Low et al. 2011; Potts \& Rothman 2008), less research has specifically evaluated factors that

252 differentiate dengue from other causes of an acute febrile illness in the adult population

253 (Chadwick et al. 2006; Gregory et al. 2010; Low et al. 2011). All but one dengue case in our

254 study presented with fever and a high percentage of cases had headache, myalgia and/or 
255 arthralgia (Chadwick et al. 2006; Hammond et al. 2005; Kittigul et al. 2007; Potts \& Rothman

256 2008). These symptoms are consistent with the previous reports of dengue in adults, but were

257 common among both dengue cases and non-dengue cases (Low et al. 2011). The only two

258 symptoms that were significantly associated with dengue in our population were red eyes and the

259 absence of a sore throat. Red eyes have not been commonly associated with dengue (Chadwick

260 et al. 2006), though one prior study found an association with DENV-1 (Yung et al. 2015). The

261 absence of a sore throat has been associated with dengue in a previous series (Gregory et al.

262 2010). However, this was only reported by $26.5 \%$ of our patients overall, which limits the utility

263 of this finding in clinical practice.

264

265 In contrast to clinical findings, the results of general laboratory studies differed significantly

266 between dengue cases and non-cases. Leucopenia and thrombocytopenia were associated with

267 dengue (ORs 11.0 and 4.0, respectively), a finding that has been consistently documented in

268 previous studies (Biswas et al. 2012; Kalayanarooj et al. 1997; Low et al. 2011). However,

269 patients with both findings did not have higher odds of dengue that those with leucopenia alone,

270 which may have resulted from temporal differences in the development and resolution of these

271 abnormalities (Biswas et al. 2012). The nadir leucocyte counts occurred on days 5-6 after

272 symptom onset, whereas platelet counts demonstrated a consistent decline through day 8 (see

273 Supplemental Files, Raw Data). Many factors were significantly associated with hospitalization

274 in univariate analyses but were also strongly correlated with one another (viral load, antibody

275 status, platelet count, day of illness). Given the sample size, our ability to model all of these

276 factors in logistic regression was limited, and admission decisions were likely based on the 
277 platelet count, which may have obscured the association between other factors and disease

278 severity.

279

280 DENV infections were confirmed using a combination of methods, though all but four cases

281 were positive by rRT-PCR $(115 / 119,96.6 \%)$. NS1 was detected in $71.4 \%$ of infections and

282 proved specific for DENV (96.4\%). Notably, the performance of this commercial NS1 kit has

283 not been published, but results appeared similar to those reported for other rapid NS1 assays

284 (Blacksell et al. 2011). Consistent with previous observations, viral loads were significantly

285 higher among NS1-positive individuals (Duong et al. 2011; Duyen et al. 2011; Erra et al. 2013;

286 Tricou et al. 2011). Both viral load and NS1 detection were significantly associated with the

287 detection of anti-DENV IgG and anti-ZIKV IgG, which in this study was directed against the

288 NS1 antigen. In an earlier study, ZIKV-specific neutralizing antibodies were not detected among

289 a subset of our patients (A. Rojas, unpublished data). As such, anti-ZIKV IgG identified by

290 ELISA in the current study is favored to represent cross-reacting anti-DENV antibodies. In the

291 subset of patients with results for both IgG assays, the presence of anti-ZIKV NS1 IgG

292 accounted for virtually all false-negative NS1 results. Although such antibodies have been

293 known to reduce NS1 detection in secondary cases (Jayathilaka et al. 2018; Lee et al. 2015; Lima

294 Mda et al. 2014), the pathophysiologic significance of anti-NS1 antibodies in human DENV

295 infections remains unclear (Glasner et al. 2018; Jayathilaka et al. 2018). We demonstrate that

296 these antibodies can be detected in the acute-phase and, in combination with anti-DENV IgG, are

297 more common among hospitalized dengue cases. These serologic findings combined with an

298 elevated DENV viral load warrant further evaluation using standardized severity criteria (World

299 Health Organization 1997; World Health Organization 2009). 
301 Dengue cases were less likely to report living in a house or apartment (recorded as "other" in the 302 study questionnaire). This was also observed in a seroprevalence study in Mexico where these 303 patients reported a "shared" living arrangement (Pavia-Ruz et al. 2018). Other aspects of the 304 home environment evaluated in our study did not differ between dengue and non-dengue cases.

305 The absence of air conditioning and window screens did not appear to increase the risk for 306 DENV infection. However, complete screening of the home and air conditioning have been 307 associated with decreased vector indices and dengue incidence in other settings (Manrique-Saide 308 et al. 2015; Pavia-Ruz et al. 2018; Reiter et al. 2003; Waterman et al. 1985), and the addition of screens has been proposed as a means of DENV control through improvements to the built environment (Lindsay et al. 2017; Vazquez-Prokopec et al. 2016). Our findings may indicate that patients acquired DENV outside the home or that the use of these interventions is incomplete (e.g. non-intact screens, intermittent use of air conditioning). Determining the location of exposure will have important implications for DENV control efforts in metro Asunción.

Vaccination against YFV is not part of the routine schedule in Paraguay, and as a result, our patient population included a mixture of individuals who did or did not report receiving the vaccine. There was no evidence of increased risk from YFV vaccination for either incident dengue or the development of severe disease. These data are consistent with recent findings from 
322 DENV-1 was the predominant serotype identified in the current study. This is consistent with

323 recent DENV epidemiology in Paraguay but precluded a comparison of symptoms caused by

324 each serotype. DENV-1 is less commonly associated with severity than DENV-2, though severe

325 and debilitating illness still occurs (Balmaseda et al. 2006; Low et al. 2011; Thomas et al. 2014).

326 Clinical findings in our patients appear more consistent with dengue in adults rather than dengue

327 caused specifically by DENV-1, which is often associated with lower rates of arthralgia and

328 myalgia (Burattini et al. 2016; Martins Vdo et al. 2014; Suppiah et al. 2018; Yung et al. 2015).

329 An additional limitation to the study is that we were unable to evaluate the performance of the

330 clinical case definition for different arboviral infections, and in particular ZIKV infections that

331 may not present with fever (Braga et al. 2017). Finally, patients were included who reported up

332 to 8 days of symptoms prior to enrollment. Laboratory data from day 8 produced conflicting

333 results and raises questions regarding the accuracy of symptom recall past one week. These data

334 support the use of earlier enrollment cut-offs with scheduled follow-up visits to monitor the

335 kinetics of certain laboratory findings.

336

337 Conclusions

338 In this study, we sought to characterize DENV infections in a predominantly adult population in

339 Paraguay, focusing on the region with the highest dengue incidence, metro Asunción. This work

340 highlighted clinical, epidemiologic, and laboratory factors that are associated with DENV

341 detection in the acute setting and the potential role of specific antibodies in diagnosis and the

342 progression of disease. Future directions will involve the prospective evaluation of how factors

343 identified in the current study associate with and may predict dengue severity. 


\section{Acknowledgements}

345 We thank the members of the study team based at the Instituto de Investigaciones en Ciencias de

346 la Salud, Universidad Nacional de Asunción, and Hospital Villa Elisa in Paraguay for their

347 dedication and excellent work, and we are grateful to the study participants and their families.

348 The authors would like to thank Diagnostic Bioprobes who kindly provided the ZIKVG.CE kits

349 used in this study as well as Muktha Natrajan and Varun Phadke for their thoughtful comments

350 during the preparation of this manuscript. 


\section{References}

Balmaseda A, Hammond SN, Perez L, Tellez Y, Saborio SI, Mercado JC, Cuadra R, Rocha J, Perez MA, Silva S, Rocha C, and Harris E. 2006. Serotype-specific differences in clinical manifestations of dengue. Am J Trop Med Hyg 74:449-456.

Bhatt S, Gething PW, Brady OJ, Messina JP, Farlow AW, Moyes CL, Drake JM, Brownstein JS, Hoen AG, Sankoh O, Myers MF, George DB, Jaenisch T, Wint GR, Simmons CP, Scott TW, Farrar JJ, and Hay SI. 2013. The global distribution and burden of dengue. Nature 496:504-507.

Biswas HH, Ortega O, Gordon A, Standish K, Balmaseda A, Kuan G, and Harris E. 2012. Early clinical features of dengue virus infection in nicaraguan children: a longitudinal analysis. PLoS Negl Trop Dis 6:e1562.

Blacksell SD, Jarman RG, Bailey MS, Tanganuchitcharnchai A, Jenjaroen K, Gibbons RV, Paris DH, Premarantna R, de Silva HJ, Lalloo DG, and Day NPJ. 2011. Evaluation of six commercial point-of-care tests for diagnosis of acute dengue infections: the need for combining NS1 antigen and IgM/IgG antibody detection to achieve acceptable levels of accuracy. Clin Vaccine Immunol 18:2095-2101.

Braga JU, Bressan C, Dalvi APR, Calvet GA, Daumas RP, Rodrigues N, Wakimoto M, Nogueira RMR, NielsenSaines K, Brito C, Bispo de Filippis AM, and Brasil P. 2017. Accuracy of Zika virus disease case definition during simultaneous Dengue and Chikungunya epidemics. PLoS One 12:e0179725.

Burattini MN, Lopez LF, Coutinho FA, Siqueira JB, Jr., Homsani S, Sarti E, and Massad E. 2016. Age and regional differences in clinical presentation and risk of hospitalization for dengue in Brazil, 2000-2014. Clinics (Sao Paulo) 71:455-463.

Chadwick D, Arch B, Wilder-Smith A, and Paton N. 2006. Distinguishing dengue fever from other infections on the basis of simple clinical and laboratory features: application of logistic regression analysis. J Clin Virol $35: 147-153$.

Dantes HG, Farfan-Ale JA, and Sarti E. 2014. Epidemiological trends of dengue disease in Mexico (2000-2011): a systematic literature search and analysis. PLoS Negl Trop Dis 8:e3158.

Dirección General de Vigilancia de la Salud, and Ministerio de Salud Pública y Bienestar Social. 2017. Boletín Epidemiológico: SE 1 (3 de enero) a SE 52 (31 de diciembre).

Duong V, Ly S, Lorn Try P, Tuiskunen A, Ong S, Chroeung N, Lundkvist A, Leparc-Goffart I, Deubel V, Vong S, and Buchy P. 2011. Clinical and virological factors influencing the performance of a NS1 antigen-capture assay and potential use as a marker of dengue disease severity. PLoS Negl Trop Dis 5:e1244.

Duyen HT, Ngoc TV, Ha do T, Hang VT, Kieu NT, Young PR, Farrar JJ, Simmons CP, Wolbers M, and Wills BA. 2011. Kinetics of plasma viremia and soluble nonstructural protein 1 concentrations in dengue: differential effects according to serotype and immune status. J Infect Dis 203:1292-1300.

Erra EO, Korhonen EM, Voutilainen L, Huhtamo E, Vapalahti O, and Kantele A. 2013. Dengue in travelers: kinetics of viremia and NS1 antigenemia and their associations with clinical parameters. PLoS One 8:e65900.

Glasner DR, Puerta-Guardo H, Beatty PR, and Harris E. 2018. The Good, the Bad, and the Shocking: The Multiple Roles of Dengue Virus Nonstructural Protein 1 in Protection and Pathogenesis. Annu Rev Virol 5:227-253.

Gordon A, Kuan G, Mercado JC, Gresh L, Aviles W, Balmaseda A, and Harris E. 2013. The Nicaraguan pediatric dengue cohort study: incidence of inapparent and symptomatic dengue virus infections, 2004-2010. PLoS Negl Trop Dis 7:e2462. 
391

392

393

394

395

396

397

398

399

400

401

402

403

404

405

406

407

408

409

410

411

412

413

414

415

416

417

418

419

420

421

422

423

424

425

426

427

428

429

430

431

Gregory CJ, Santiago LM, Arguello DF, Hunsperger E, and Tomashek KM. 2010. Clinical and laboratory features that differentiate dengue from other febrile illnesses in an endemic area--Puerto Rico, 2007-2008. Am J Trop Med Hyg 82:922-929.

Guzman MG, and Harris E. 2015. Dengue. Lancet 385:453-465.

Halsey ES, Marks MA, Gotuzzo E, Fiestas V, Suarez L, Vargas J, Aguayo N, Madrid C, Vimos C, Kochel TJ, and Laguna-Torres VA. 2012. Correlation of serotype-specific dengue virus infection with clinical manifestations. PLoS Negl Trop Dis 6:e1638.

Hammond SN, Balmaseda A, Perez L, Tellez Y, Saborio SI, Mercado JC, Videa E, Rodriguez Y, Perez MA, Cuadra R, Solano S, Rocha J, Idiaquez W, Gonzalez A, and Harris E. 2005. Differences in Dengue severity in infants, children, and adults in a 3-year hospital-based study in Nicaragua. Am J Trop Med Hyg 73:1063.

Jayathilaka D, Gomes L, Jeewandara C, Jayarathna GSB, Herath D, Perera PA, Fernando S, Wijewickrama A, Hardman CS, Ogg GS, and Malavige GN. 2018. Role of NS1 antibodies in the pathogenesis of acute secondary dengue infection. Nat Commun 9:5242.

Kalayanarooj S, Vaughn DW, Nimmannitya S, Green S, Suntayakorn S, Kunentrasai N, Viramitrachai W, Ratanachu-eke S, Kiatpolpoj S, Innis BL, Rothman AL, Nisalak A, and Ennis FA. 1997. Early clinical and laboratory indicators of acute dengue illness. J Infect Dis 176:313-321.

Kittigul L, Pitakarnjanakul P, Sujirarat D, and Siripanichgon K. 2007. The differences of clinical manifestations and laboratory findings in children and adults with dengue virus infection. J Clin Virol 39:76-81.

Lee J, Kim HY, Chong CK, and Song HO. 2015. Development and clinical evaluation of a highly accurate dengue NS1 rapid test: from the preparation of a soluble NS1 antigen to the construction of an RDT. Diagn Microbiol Infect Dis 82:128-134.

Lima Mda R, Nogueira RM, Filippis AM, Nunes PC, Sousa CS, Silva MH, and Santos FB. 2014. A simple heat dissociation method increases significantly the ELISA detection sensitivity of the nonstructural-1 glycoprotein in patients infected with DENV type-4. J Virol Methods 204:105-108.

Lindsay SW, Wilson A, Golding N, Scott TW, and Takken W. 2017. Improving the built environment in urban areas to control Aedes aegypti-borne diseases. Bull World Health Organ 95:607-608.

Lovera D, Araya S, Mesquita MJ, Avalos C, Ledesma S, and Arbo A. 2014. Prospective applicability study of the new dengue classification system for clinical management in children. Pediatr Infect Dis J 33:933-935.

Lovera D, Martinez de Cuellar C, Araya S, Amarilla S, Gonzalez N, Aguiar C, Acuna J, and Arbo A. 2016. Clinical Characteristics and Risk Factors of Dengue Shock Syndrome in Children. Pediatr Infect Dis J 35:12941299.

Low JG, Ong A, Tan LK, Chaterji S, Chow A, Lim WY, Lee KW, Chua R, Chua CR, Tan SW, Cheung YB, Hibberd ML, Vasudevan SG, Ng LC, Leo YS, and Ooi EE. 2011. The early clinical features of dengue in adults: challenges for early clinical diagnosis. PLoS Negl Trop Dis 5:e1191.

Luppe MJ, Verro AT, Barbosa AS, Nogueira ML, Undurraga EA, and da Silva NS. 2019. Yellow fever (YF) vaccination does not increase dengue severity: A retrospective study based on 11,448 dengue notifications in a YF and dengue endemic region. Travel Med Infect Dis.

Manrique-Saide P, Che-Mendoza A, Barrera-Perez M, Guillermo-May G, Herrera-Bojorquez J, Dzul-Manzanilla F, Gutierrez-Castro C, Lenhart A, Vazquez-Prokopec G, Sommerfeld J, McCall PJ, Kroeger A, and Arredondo-Jimenez JI. 2015. Use of insecticide-treated house screens to reduce infestations of dengue virus vectors, Mexico. Emerg Infect Dis 21:308-311.

Peer] reviewing PDF | (2019:06:38824:1:2:NEW 29 Aug 2019) 
432

433

434

435

436

437

438

439

440

441

442

443

444

445

446

447

448

449

450

451

452

453

454

455

456

457

458

459

460

461

462

463

464

465

466

467

468

469

470

471

472

473

474

Martins Vdo C, Bastos Mde S, Ramasawmy R, de Figueiredo RP, Gimaque JB, Braga WS, Nogueira ML, Nozawa S, Naveca FG, Figueiredo LT, and Mourao MP. 2014. Clinical and virological descriptive study in the 2011 outbreak of dengue in the Amazonas, Brazil. PLoS One 9:e100535.

Morch K, Manoharan A, Chandy S, Chacko N, Alvarez-Uria G, Patil S, Henry A, Nesaraj J, Kuriakose C, Singh A, Kurian S, Gill Haanshuus C, Langeland N, Blomberg B, Vasanthan Antony G, and Mathai D. 2017. Acute undifferentiated fever in India: a multicentre study of aetiology and diagnostic accuracy. BMC Infect Dis $17: 665$.

O. Silva MM, Tauro LB, Kikuti M, Anjos RO, Santos VC, Goncalves TSF, Paploski IAD, Moreira PSS, Nascimento LCJ, Campos GS, Ko AI, Weaver SC, Reis MG, Kitron U, and Ribeiro GS. 2018. Concomitant transmission of dengue, chikungunya and Zika viruses in Brazil: Clinical and epidemiological findings from surveillance for acute febrile illness. Clin Infect Dis.

Pan American Health Organization. 2018. Dengue. Available at https: $/ / w w w . p a h o . o r g / h q /$ index.php? option $=$ com topics \&view $=$ article $\&$ id $=1 \&$ Itemid $=40734 \&$ lang $=e n$ (accessed 15 May 2018.

Pavia-Ruz N, Barrera-Fuentes GA, Villanueva-Jorge S, Che-Mendoza A, Campuzano-Rincon JC, Manrique-Saide P, Rojas DP, Vazquez-Prokopec GM, Halloran ME, Longini IM, and Gomez-Dantes H. 2018. Dengue seroprevalence in a cohort of schoolchildren and their siblings in Yucatan, Mexico (2015-2016). PLoS Negl Trop Dis 12:e0006748.

Peeling RW, Artsob H, Pelegrino JL, Buchy P, Cardosa MJ, Devi S, Enria DA, Farrar J, Gubler DJ, Guzman MG, Halstead SB, Hunsperger E, Kliks S, Margolis HS, Nathanson CM, Nguyen VC, Rizzo N, Vazquez S, and Yoksan S. 2010. Evaluation of diagnostic tests: dengue. Nat Rev Microbiol 8:S30-38.

Perez F, Llau A, Gutierrez G, Bezerra H, Coelho G, Ault S, Barbiratto SB, de Resende MC, Cerezo L, Kleber GL, Pacheco O, Perez OL, Picos V, Rojas DP, Siqueira JB, Suarez MF, Harris E, Castellanos LG, Espinal C, and Martin JLS. 2019. The decline of dengue in the Americas in 2017: discussion of multiple hypotheses. Trop Med Int Health.

Potts JA, and Rothman AL. 2008. Clinical and laboratory features that distinguish dengue from other febrile illnesses in endemic populations. Trop Med Int Health 13:1328-1340.

Reiter P, Lathrop S, Bunning M, Biggerstaff B, Singer D, Tiwari T, Baber L, Amador M, Thirion J, Hayes J, Seca C, Mendez J, Ramirez B, Robinson J, Rawlings J, Vorndam V, Waterman S, Gubler D, Clark G, and Hayes E. 2003. Texas lifestyle limits transmission of dengue virus. Emerg Infect Dis 9:86-89.

Rojas A, Aria L, de Guillen YA, Acosta ME, Infanzón B, Diaz V, López L, Meza T, and Riveros O. 2016. Perfil clínico, hematológico y serológico en pacientes con sospecha de dengue del IICS-UNA, 2009-2013. Mem Inst Investig Cienc Salud 14:68-74.

Stanaway JD, Shepard DS, Undurraga EA, Halasa YA, Coffeng LE, Brady OJ, Hay SI, Bedi N, Bensenor IM, Castaneda-Orjuela CA, Chuang TW, Gibney KB, Memish ZA, Rafay A, Ukwaja KN, Yonemoto N, and Murray CJ. 2016. The global burden of dengue: an analysis from the Global Burden of Disease Study 2013. Lancet Infect Dis 16:712-723.

Suppiah J, Ching SM, Amin-Nordin S, Mat-Nor LA, Ahmad-Najimudin NA, Low GK, Abdul-Wahid MZ, Thayan R, and Chee HY. 2018. Clinical manifestations of dengue in relation to dengue serotype and genotype in Malaysia: A retrospective observational study. PLoS Negl Trop Dis 12:e0006817.

Thomas L, Najioullah F, Besnier F, Valentino R, Cesaire R, Rosine J, Cabie A, and Working Group on D. 2014. Clinical presentation of dengue by serotype and year of epidemic in Martinique. Am J Trop Med Hyg 91:138-145.

Peer] reviewing PDF | (2019:06:38824:1:2:NEW 29 Aug 2019) 
475 476

477

478

479

480

481

482

483

484

485

486

487

488

489

490

491

492

493

494

495

496

497

498

499

500

501

502

503

504

505

506

507
Tricou V, Minh NN, Farrar J, Tran HT, and Simmons CP. 2011. Kinetics of viremia and NS1 antigenemia are shaped by immune status and virus serotype in adults with dengue. PLoS Negl Trop Dis 5:e1309.

Vazquez-Prokopec GM, Lenhart A, and Manrique-Saide P. 2016. Housing improvement: a novel paradigm for urban vector-borne disease control? Trans R Soc Trop Med Hyg 110:567-569.

Waggoner JJ, Abeynayake J, Sahoo MK, Gresh L, Tellez Y, Gonzalez K, Ballesteros G, Balmaseda A, Karunaratne K, Harris E, and Pinsky BA. 2013a. Development of an internally controlled real-time reverse transcriptase PCR assay for pan-dengue virus detection and comparison of four molecular dengue virus detection assays. J Clin Microbiol 51:2172-2181.

Waggoner JJ, Abeynayake J, Sahoo MK, Gresh L, Tellez Y, Gonzalez K, Ballesteros G, Guo FP, Balmaseda A, Karunaratne K, Harris E, and Pinsky BA. 2013b. Comparison of the FDA-approved CDC DENV-1-4 realtime reverse transcription-PCR with a laboratory-developed assay for dengue virus detection and serotyping. J Clin Microbiol 51:3418-3420.

Waggoner JJ, Abeynayake J, Sahoo MK, Gresh L, Tellez Y, Gonzalez K, Ballesteros G, Pierro AM, Gaibani P, Guo FP, Sambri V, Balmaseda A, Karunaratne K, Harris E, and Pinsky BA. 2013c. Single-reaction, multiplex, real-time rt-PCR for the detection, quantitation, and serotyping of dengue viruses. PLoS Negl Trop Dis 7:e2116.

Waggoner JJ, Gresh L, Mohamed-Hadley A, Ballesteros G, Davila MJ, Tellez Y, Sahoo MK, Balmaseda A, Harris E, and Pinsky BA. 2016a. Single-Reaction Multiplex Reverse Transcription PCR for Detection of Zika, Chikungunya, and Dengue Viruses. Emerg Infect Dis 22:1295-1297.

Waggoner JJ, Gresh L, Vargas MJ, Ballesteros G, Tellez Y, Soda KJ, Sahoo MK, Nunez A, Balmaseda A, Harris E, and Pinsky BA. 2016b. Viremia and Clinical Presentation in Nicaraguan Patients Infected With Zika Virus, Chikungunya Virus, and Dengue Virus. Clin Infect Dis 63:1584-1590.

Waterman SH, Novak RJ, Sather GE, Bailey RE, Rios I, and Gubler DJ. 1985. Dengue transmission in two Puerto Rican communities in 1982. Am J Trop Med Hyg 34:625-632.

World Health Organization. 1997. Dengue hemorrhagic fever: diagnosis, treatment, prevention and control. Geneva: World Health Organization.

World Health Organization. 2009. Dengue: guidelines for diagnosis, treatment, prevention and control. France: WHO Press.

Yung CF, Lee KS, Thein TL, Tan LK, Gan VC, Wong JGX, Lye DC, Ng LC, and Leo YS. 2015. Dengue serotypespecific differences in clinical manifestation, laboratory parameters and risk of severe disease in adults, singapore. Am J Trop Med Hyg 92:999-1005. 


\section{Figure Legends}

509 Figure 1. Map of South America highlighting Paraguay and surrounding countries. Inset tables

510 show the dengue serotypes reported by each country to the Pan American Health Organization

511 for the years 2014-2018 (data obtained from paho.org, accessed 16 August 2019). The included

512 countries are shaded from dark to light blue according to the number of circulating DENV

513 serotypes identified during this period (generated at mapchart.net under the license CC BY-SA

514 4.0).

515

516 Figure 2. Sensitivity of rRT-PCR, NS1, and IgM for dengue based on day of illness at

517 presentation.

518

519 Figure 3. DENV-1 viral load by day of illness at presentation (A). Viral loads are shown for 520 individual samples; bars display the mean and 95\% CI. Six patients had infections with DENV-2 $521(n=3)$ or DENV-4 $(n=3)$, which are not displayed. DENV viral load at presentation decreases in a 522 stepwise manner among individuals with anti-ZIKV IgG, anti-DENV IgG, or both (B). Results 523 were significant by ANOVA for both analyses, $\mathrm{p}<0.0001$.

524

525 Figure 4. Platelet (A) and leucocyte (B) counts at presentation among dengue cases (•) and non526 dengue cases $(\triangle)$. Bars represent means $\pm 95 \% \mathrm{CI}$; population mean values are shown. 


\section{Table 1 (on next page)}

DENV diagnostic test results according to test method. DENV viral load is shown for rRTPCR positive samples within a given category. 
1 Table 1. DENV diagnostic test results according to test method. DENV viral load is shown for

2 rRT-PCR positive samples within a given category.

\begin{tabular}{l|cc|cc} 
& \multicolumn{2}{|c|}{ Composite Definition } & & \\
Test Results & Positive & Negative & Day of Illness & Viral Load \\
\hline Combination of Methods & $(\mathrm{n}=119)$ & $(\mathrm{n}=112)$ & mean (sd) & mean (sd) ${ }^{\mathrm{a}}$ \\
rRT-PCR & $28(23.5)$ & - & $3.1(1.5)$ & $6.10(1.69)$ \\
rRT-PCR and NS1 & $60(50.4)$ & - & $3.2(1.4)$ & $7.85(1.27)$ \\
rRT-PCR, NS1, and IgM & $21(17.7)$ & - & $5.3(1.3)$ & $5.40(1.19)$ \\
rRT-PCR and IgM & $6(5.0)$ & - & $6.7(1.0)$ & $3.58(0.22)$ \\
NS1 and IgM & $4(3.4)$ & - & $7.2(0.1)$ & - \\
Negative & - & 112 & $3.2(1.6)$ & - \\
Positives according to method & & & & \\
rRT-PCR & $115(96.6)$ & - & $3.7(1.7)$ & \\
NS1 & $85(71.4)$ & $4(3.6)^{\mathrm{b}}$ & $3.9(1.8)$ & \\
IgM & $31(26.1)$ & $7(6.2)^{\mathrm{b}}$ & $5.6(1.5)$ & \\
\hline
\end{tabular}

$3 \quad{ }^{a}$ Reported as $\log _{10}$ copies/mL of serum

$4 \quad \mathrm{~b}$ Specificities were $96.4 \%(\mathrm{NS} 1)$ and $93.8 \%(\operatorname{IgM})$ 
Table 2 (on next page)

Epidemiologic data on patients presenting with an acute febrile illness who tested positive or negative for DENV. 
1 Table 2. Epidemiologic data on patients presenting with an acute febrile illness who tested

2 positive or negative for DENV.

\begin{tabular}{|c|c|c|c|c|}
\hline Factor ${ }^{\text {a }}$ & Total & Dengue Cases & Non-Dengue & p-value \\
\hline Patients & $231(100)$ & $119(100)$ & $112(100)$ & \\
\hline Gender, female & $128(55.4)$ & $63(52.9)$ & $65(58.0)$ & \\
\hline Age, mean (sd) & $31.94(14.3)$ & $31.3(15.0)$ & $32.6(13.6)$ & \\
\hline \multicolumn{5}{|l|}{ Clinical Site } \\
\hline Hospital Villa Elisa & $185(80.1)$ & $100(84.0)$ & $85(75.9)$ & \\
\hline IICS-UNA & $46(19.9)$ & $19(16.0)$ & $27(24.1)$ & \\
\hline \multicolumn{5}{|l|}{ Department } \\
\hline Central & $209(90.5)$ & $109(91.6)$ & $100(89.3)$ & \\
\hline Capital & $20(8.7)$ & $8(6.7)$ & $12(10.7)$ & \\
\hline \multicolumn{5}{|l|}{ Residence } \\
\hline House & $149(79.3)$ & $70(74.4)$ & $79(84.0)$ & \\
\hline Apartment & $8(4.3)$ & $2(2.1)$ & $6(6.4)$ & \\
\hline Other & $31(16.5)$ & $22(23.4)$ & $9(9.6)$ & 0.017 \\
\hline Screens & $21(10.6)$ & $10(10.0)$ & $11(11.1)$ & \\
\hline Air-conditioning & $156(79.2)$ & $76(78.4)$ & $80(80.0)$ & \\
\hline Running water & $199(98.0)$ & $97(97.0)$ & $102(99.0)$ & \\
\hline Water storage & $18(8.8)$ & $8(8.0)$ & $10(9.5)$ & \\
\hline \multicolumn{5}{|l|}{ Exposures } \\
\hline Travel in the last month & $52(25.0)$ & $22(20.8)$ & $30(29.4)$ & \\
\hline Work or school outside of the home & $152(80.4)$ & $68(73.9)$ & $84(86.6)$ & \\
\hline Work or school outdoors & $24(55.8)$ & $11(57.9)$ & $13(54.2)$ & \\
\hline \multicolumn{5}{|l|}{ Medical History } \\
\hline Received yellow fever vaccine & $71(42.0)$ & $31(37.3)$ & $40(46.5)$ & \\
\hline Years since vaccination, mean $(\mathrm{sd})^{\mathrm{c}}$ & $7.6(4.1)$ & $8.5(3.7)$ & $6.8(4.3)$ & \\
\hline
\end{tabular}


3 Abbreviations: $n$, number; sd, standard deviation

$4{ }^{a}$ Unless otherwise specific, all values presented as $\mathrm{n}(\%$ of patients with a response recorded)

5 b OR for dengue for patients reporting “other", 2.9 (95\% CI 1.3-7.0)

$6{ }^{c}$ Year of YF vaccination was available for 52 patients (24 DENV-positive, 28 DENV-negative)

$7{ }^{\mathrm{d}} \mathrm{OR}$ for dengue in patients who reported a history of dengue, 0.4 (95\% CI 0.2-0.7) 


\section{Table 3 (on next page)}

Symptoms and laboratory findings among patients with and without dengue. 
1 Table 3. Symptoms and laboratory findings among patients with and without dengue.

\begin{tabular}{|c|c|c|c|c|c|}
\hline Factor a & Total & Dengue Cases & Non-Dengue & OR $(95 \% \text { CI })^{b}$ & p-value \\
\hline Patients & $231(100)$ & $119(100)$ & $112(100)$ & & \\
\hline Day of symptoms, mean (sd) & $3.9(2.5)$ & $4.1(1.9)$ & $3.7(3.0)$ & & \\
\hline \multicolumn{6}{|l|}{ Symptoms and signs at presentation } \\
\hline Fever & $221(96.5)$ & $117(99.2)$ & $104(93.7)$ & & \\
\hline Headache & $206(89.6)$ & $111(93.3)$ & $95(85.6)$ & $2.3(1.0-5.5)$ & 0.083 \\
\hline Retro-orbital pain & $94(40.9)$ & $53(44.5)$ & $41(36.9)$ & & \\
\hline Muscle pain & $198(88.0)$ & $99(86.8)$ & $99(89.2)$ & & \\
\hline Joint pain & $172(77.8)$ & $92(80.7)$ & $80(74.8)$ & & \\
\hline Nausea & $142(61.7)$ & $73(61.3)$ & $69(62.2)$ & & \\
\hline Malaise & $119(51.7)$ & $62(52.1)$ & $57(51.4)$ & & \\
\hline Red eyes & $99(45.0)$ & $61(51.5)$ & $38(35.8)$ & $2.1(1.2-3.6)$ & 0.010 \\
\hline Abdominal pain & $95(41.3)$ & $51(42.9)$ & $44(39.6)$ & & \\
\hline Vomiting & $73(31.7)$ & $41(34.5)$ & $32(28.8)$ & & \\
\hline Diarrhea & $66(28.7)$ & $31(26.1)$ & $35(31.5)$ & & \\
\hline Shortness of breath & $64(27.8)$ & $36(30.3)$ & $28(25.2)$ & & \\
\hline Sore throat & $61(26.5)$ & $23(19.3)$ & $38(34.2)$ & $0.5(0.3-0.8)$ & 0.011 \\
\hline Cough & $51(22.2)$ & $25(21.0)$ & $26(23.4)$ & & \\
\hline Rash & $52(23.1)$ & $32(27.8)$ & $20(18.2)$ & & \\
\hline Edema & $37(16.2)$ & $17(14.3)$ & $20(18.0)$ & & \\
\hline Bleeding & $32(13.9)$ & $20(16.8)$ & $12(10.8)$ & & \\
\hline \multicolumn{6}{|l|}{ Laboratory results } \\
\hline Hemoglobin, g/dL, mean (sd) & $13.9(1.5)$ & $14.0(1.5)$ & $13.8(1.5)$ & & \\
\hline Platelet count, per $\mu \mathrm{L}$, mean (sd) & $217,550(89,921)$ & $188,227(82,079)$ & $252,609(86,650)$ & & $<0.001$ \\
\hline Thrombocytopenia, $<150,000$ per $\mu \mathrm{L}$ & $46(22.8)$ & $36(32.7)$ & $10(10.9)$ & $4.0(1.9-8.2)$ & $<0.001$ \\
\hline Leucocyte count, cells per $\mathrm{mm}^{3}$, mean (sd) & $6090(3686)$ & $4158(2023)$ & $8401(3899)$ & & $<0.001$ \\
\hline Leucopenia, $<4,000$ cells per $\mathrm{mm}^{3}$ & $73(36.1)$ & $63(57.3)$ & $10(10.9)$ & $11.0(5.1-22.2)$ & $<0.001$ \\
\hline
\end{tabular}

2 Abbreviations: CI confidence interval; OR, odds ratio; sd, standard deviation 
3 a Values presented as n (\%) unless otherwise indicated, percentages were calculated based on the

4 number of patients with data recorded for a particular variable

$5 \quad \mathrm{~b}$ OR of having a dengue case versus a non-dengue case 


\section{Table 4 (on next page)}

Clinical history and test results among hospitalized and outpatient dengue cases at Hospital Villa Elisa. 
1 Table 4. Clinical history and test results among hospitalized and outpatient dengue cases at

\section{Hospital Villa Elisa.}

\begin{tabular}{|c|c|c|c|c|c|}
\hline Patient Factors a & Total & Hospitalized & Outpatient & OR $(95 \% \text { CI })^{b}$ & p-value \\
\hline Patients & $100(100)$ & $26(100)$ & $74(100)$ & & \\
\hline \multicolumn{6}{|l|}{ History and Clinical findings } \\
\hline Gender, female, n (\%) & $52(52.0)$ & $12(46.2)$ & $40(54.1)$ & & \\
\hline Age, mean (sd) & $31.6(14.5)$ & $36.5(20.0)$ & $29.9(11.6)$ & & 0.044 \\
\hline Day of illness & $3.81(1.84)$ & $5.0(2.4)$ & $3.4(1.4)$ & & $<0.001$ \\
\hline YFV vaccination & $23 / 64(35.9)$ & $5 / 16(31.2)$ & $17 / 48(35.4)$ & & \\
\hline Past dengue, per report & $34 / 99(34.3)$ & $12 / 25(48.0)$ & $22 / 74(28.6)$ & $2.2(0.9-5.5)$ & 0.143 \\
\hline Rash & $28 / 96(29.2)$ & $13 / 25(52.0)$ & $15 / 71(21.1)$ & $4.0(1.5-10.0)$ & 0.005 \\
\hline Diarrhea & $27 / 100(27.0)$ & $11 / 26(42.3)$ & $16 / 74(21.6)$ & $2.7(1.0-6.9)$ & 0.070 \\
\hline Bleeding & $18 / 100(18.0)$ & $10 / 26(38.5)$ & $8 / 74(10.8)$ & $5.2(1.8-14.1)$ & 0.006 \\
\hline \multicolumn{6}{|l|}{ Dengue test results } \\
\hline rRT-PCR, positive & $99(99.0)$ & $25(96.2)$ & $74(100)$ & & \\
\hline Viral load, mean (sd) & $6.44(2.04)$ & $6.76(1.84)$ & $5.51(2.35)$ & & 0.028 \\
\hline NS1 & $69(69.0)$ & $17(65.4)$ & $52(78.4)$ & $0.8(0.3-2.0)$ & 0.632 \\
\hline IgM, anti-DENV & $25(25.0)$ & $10(38.5)$ & $15(20.3)$ & $2.5(1.0-6.6)$ & 0.112 \\
\hline IgG, anti-DENV & $28(28.0)$ & $14(53.9)$ & $14(18.9)$ & $5.0(1.9-12.2)$ & 0.002 \\
\hline IgG, anti-ZIKV & $19 / 70(27.1)$ & $7 / 16(43.8)$ & $12 / 54(22.2)$ & $2.7(0.9-8.1)$ & 0.114 \\
\hline IgG against both DENV and ZIKV & $13 / 67(19.4)$ & $7 / 13(53.8)$ & $6 / 54(11.1)$ & $9.3(2.2-36.3)$ & 0.002 \\
\hline \multicolumn{6}{|l|}{ Laboratory results ${ }^{c}$} \\
\hline Hemoglobin, g/dL, mean (sd) & $14.1(1.4)$ & $14.0(2.0)$ & $14.2(1.2)$ & & \\
\hline Platelet count, per $\mu \mathrm{L}$, mean (sd) & $191,563(85,951)$ & $119,250(77,402)$ & $215,667(74,749)$ & & $<0.001$ \\
\hline Thrombocytopenia, $<150,000$ per $\mu \mathrm{L}$ & $31(32.3)$ & $18(75.0)$ & $13(18.1)$ & $13.6(4.5-43.2)$ & $<0.001$ \\
\hline Leucocyte count, cells per $\mathrm{mm}^{3}$, mean (sd) & $4167(2135)$ & $4814(3209)$ & $3952(1604)$ & & 0.087 \\
\hline Leucopenia, $<4,000$ cells per $\mathrm{mm}^{3}$ & $55(57.3)$ & $13(54.2)$ & $42(58.3)$ & & \\
\hline
\end{tabular}

3 Abbreviations: CI confidence interval; OR, odds ratio; sd, standard deviation

$4 \quad$ a Values presented as n (\%) unless otherwise indicated

$5 \quad$ b OR for hospitalization versus outpatient care 
$6 \quad{ }^{\mathrm{c}}$ Lab results were available for 24 and 72 hospitalized cases and outpatients, respectively. 
Figure 1

Map of South America highlighting Paraguay and surrounding countries.

Inset tables show the dengue serotypes reported by each country to the Pan American Health Organization for the years 2014-2018 (data obtained from paho.org, accessed 16 August 2019). The included countries are shaded from dark to light blue according to the number of circulating DENV serotypes identified during this period (generated at mapchart.net). 


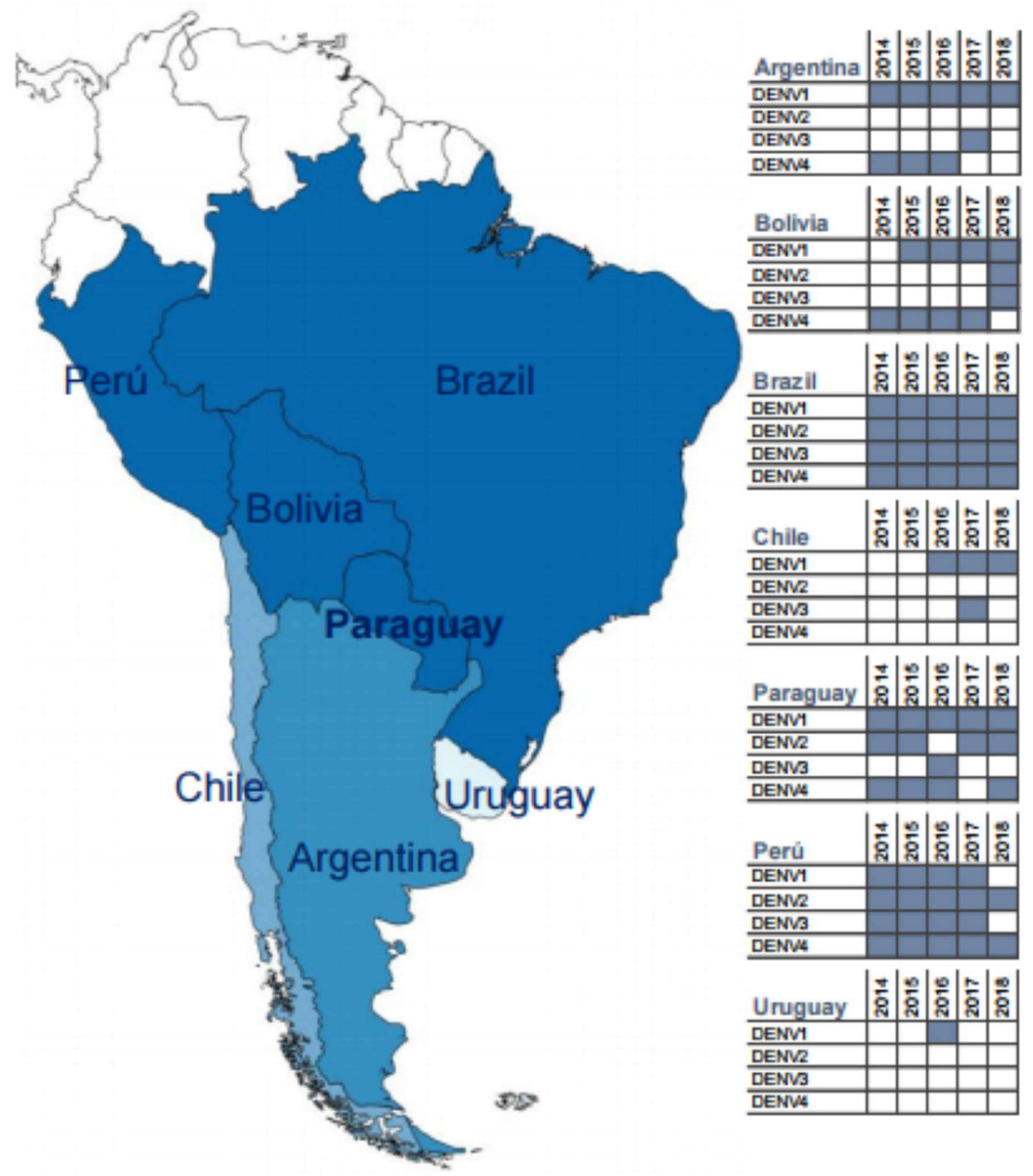


Figure 2

Sensitivity of rRT-PCR, NS1, and IgM for dengue based on day of illness at presentation.

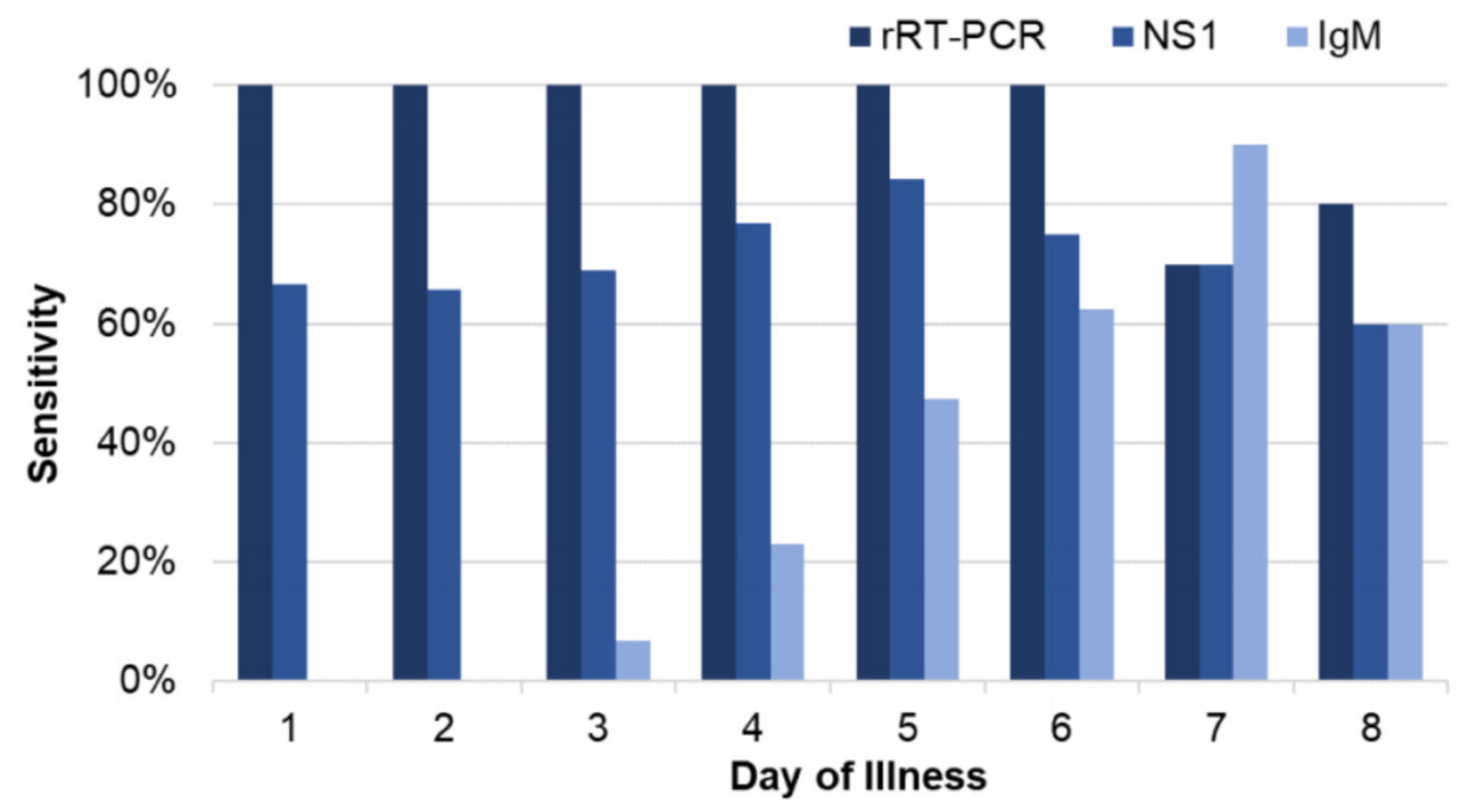


Figure 3

DENV viral load at presentation based on day of illness and antibody status.

DENV-1 viral load by day of illness at presentation (A). Viral loads are shown for individual samples; bars display the mean and $95 \% \mathrm{Cl}$. Six patients had infections with DENV-2 $(n=3)$ or DENV-4 $(n=3)$, which are not displayed. DENV viral load at presentation decreases in a stepwise manner among individuals with anti-ZIKV IgG, anti-DENV IgG, or both (B). Results were significant by ANOVA for both analyses, $p<0.0001$.
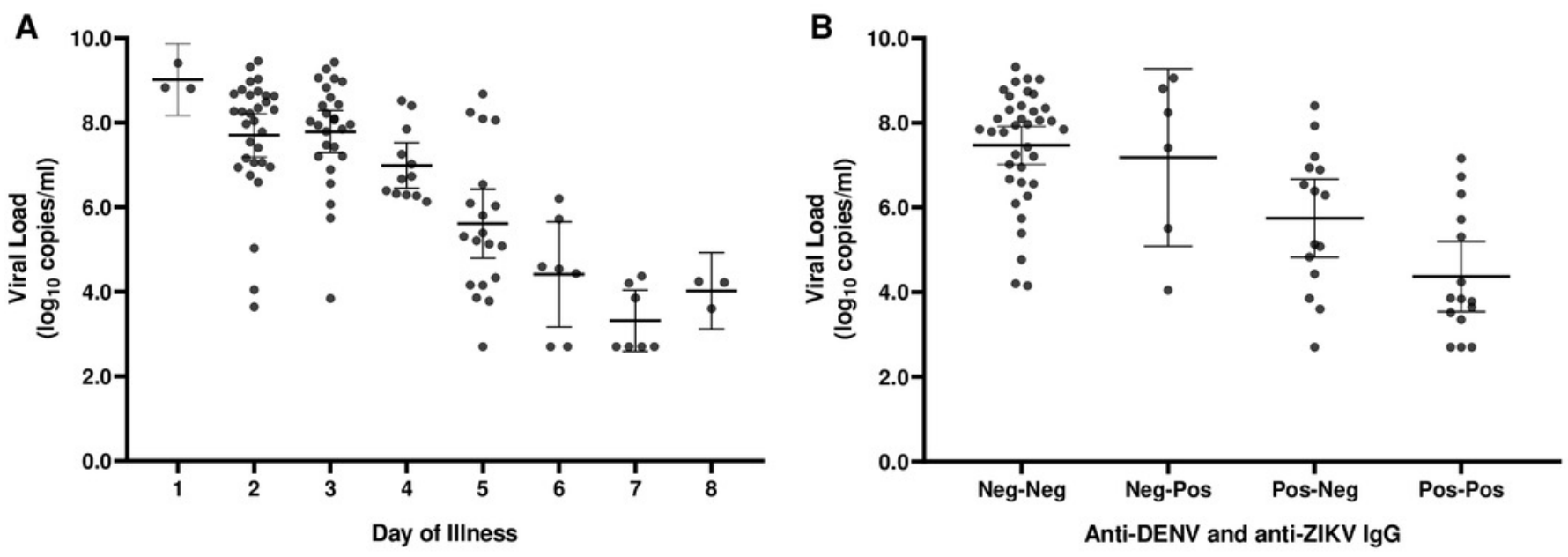
Figure 4

Platelet $(A)$ and leucocyte $(B)$ counts at presentation among dengue cases $(\mathbf{O})$ and nondengue cases $(\boldsymbol{\Lambda})$.

Bars represent means $\pm 95 \% \mathrm{Cl}$; population mean values are shown.
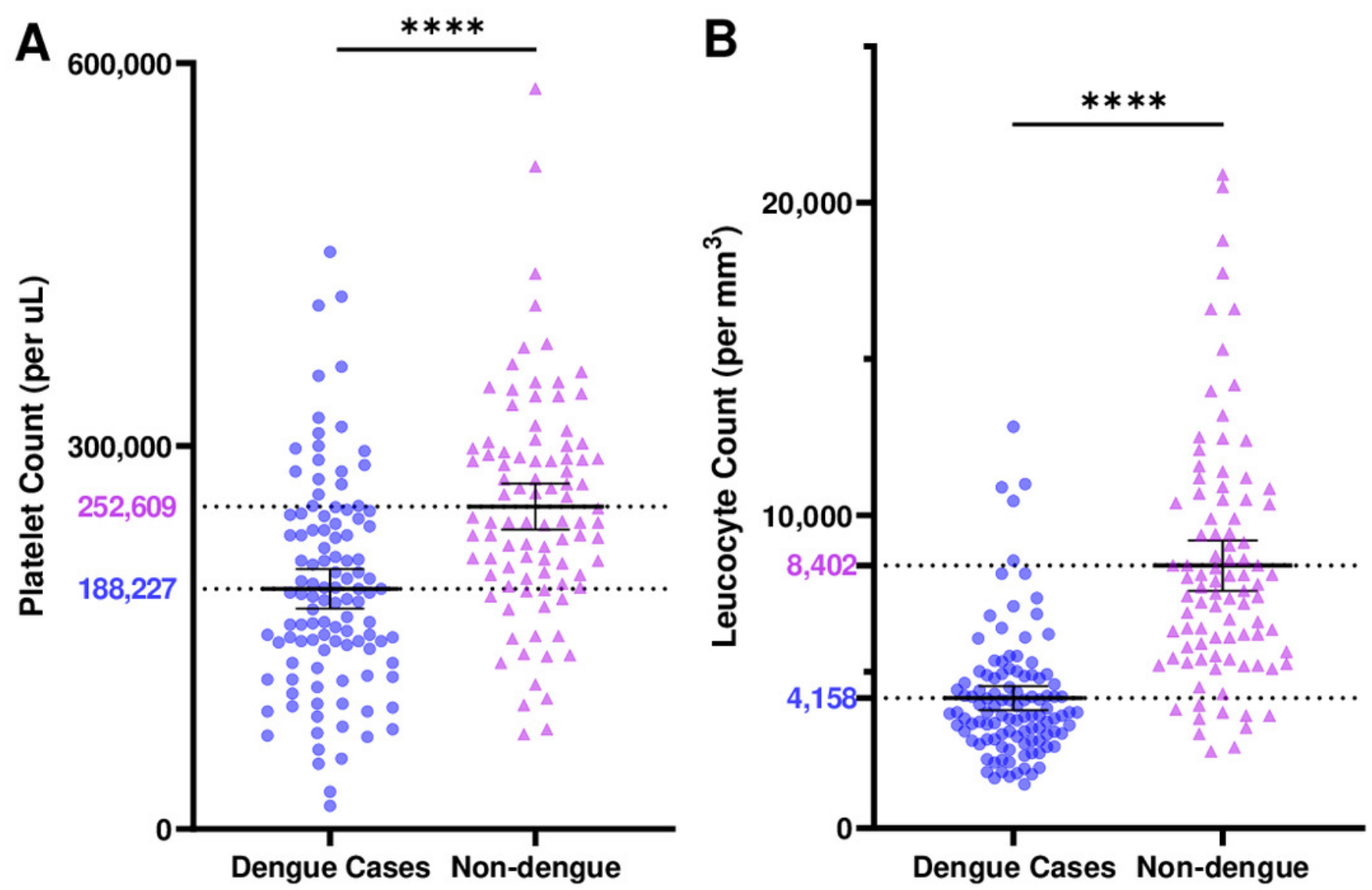